\title{
Chemistry problem solving instruction: a comparison of three computer-based formats for learning from hierarchical network problem representations
}

\author{
Bing Hiong Ngu $\cdot$ Edwin Mit · Faaizah Shahbodin • \\ Juhani Tuovinen
}

Received: 1 November 2006/Accepted: 21 November 2006/Published online: 23 September 2008

(C) Springer Science+Business Media B.V. 2008

\begin{abstract}
Within the cognitive load theory framework, we designed and compared three alternative instructional solution formats that can be derived from a common static hierarchical network representation depicting problem structure. The interactive-solution format permitted students to search in self-controlled manner for solution steps, staticsolution format displayed all solutions steps, and no-solution format did not have solution steps. When we matched instructional time across the formats, in relation to the complex molarity problems rather than the dilution problems, differential transfer performance existed between the static-solution or no-solution formats and the interactive-solution format, but not between the static-solution format and no-solution format. The manner in which learners interact with the static-solution and no-solution formats depends on their level of expertise in the chemistry domain. With considerable learner expertise, provision of solution steps may be redundant incurring extraneous cognitive load. Absence of the solution steps may not have left sufficient cognitive capacity for germane cognitive load as some beginning learners lacked the prior knowledge to deduce the solution steps. Searching for solution steps presumably incurred extraneous cognitive load which interfered with learning and hence, in the interactive-solution format, it outweighed the benefit of engaging in self-regulated interaction with the content. Hence, cognitive load theory is
\end{abstract}

\footnotetext{
B. H. Ngu

Learning and Teaching Centre, Tabor Adelaide, 181 Goodwood Road,

Millswood, SA 5034, Australia

e-mail: Bngu@adelaide.tabor.edu.au

E. Mit $\cdot$ F. Shahbodin

Faculty of Cognitive Sciences and Human Development, Universiti Malaysia Sarawak,

Kota Samarahan, Sarawak, Malaysia

e-mail: edwin@fit.unimas.my

J. Tuovinen $(\bowtie)$

Faculty of Science, Health and Education, University of the Sunshine Coast, Maroochydore,

DC 4558, Australia

e-mail: juhani.tuovinen@usc.edu.au
} 